UNIVERSIDADE FEDERAL DO RIO GRANDE DO SUL INSTITUTO DE MATEMATICA

CADERNOS DE MATEMÁTICA E ESTATISTICA

SERIE A: TRABALHO DE PESQUISA

ON THE SPECTRAL DENSITY OF A CLASS OF CHAOTIC TIME SERIES

\author{
ARTUR OSCAR LOPES \\ RAF.AEL RIGÄO SOUZA \\ SILVIA REGINA COSTA LOPES
}

SÉRIE $\triangle N^{\circ} 43$

PORTO ALEGRE, DEZHMBRO DE 1995 


\title{
ON THE SPECTRAL DENSITY OF A CLASS OF CHAOTIC TIME SERIES
}

\author{
by Artur Lopes, Sílvia Lopes and Rafael R. Souza
}

\section{Instituto de Matemática - UFRGS \\ Porto Alegre - RS - Brazil}

Abstract: The purpose of this paper is to show explicitly the spectral density of the stationary stochastic process determined by the map $F_{\alpha}$ ( $\alpha$ is a parameter in $(0,1)$ ), the random variable $\phi(x, y)=x$ and the invariant probability $\nu$ described below.

We first define the transformation $T_{\alpha}:[0,1] \rightarrow[0,1]$ given by

$$
T_{\alpha}(x)=\left\{\begin{array}{lll}
\frac{x}{\alpha}, & \text { if } & 0 \leq x<\alpha \\
\frac{\alpha(x-\alpha)}{1-\alpha}, & \text { if } & \alpha \leq x \leq 1,
\end{array}\right.
$$

where $\alpha \in(0,1)$ is a constant. The map $T_{\alpha}$ describes a model for a particle (or the probability of a certain kind of element in a given population) that moves around, in discrete time, in the interval $[0,1]$.

The map $F_{\alpha}$ is defined from $K=([0,1] \times(0, \alpha)) \cup([0, \alpha] \times[\alpha, 1])$ to itself and it is given by $F_{\alpha}(x, y)=\left(T_{\alpha}(x), G_{\alpha}(x, y)\right)$, for $(x, y) \in K$ where

$$
G_{\alpha}(x, y)= \begin{cases}\alpha y & , 0 \leq x<\alpha \\ \alpha+\left(\frac{1-\alpha}{\alpha}\right) y & , \quad \alpha \leq x \leq 1\end{cases}
$$

The spectral density function of the stationary process with probability $\nu$ (invariant and absolutely continuous measure with respect to the Lebesgue measure)

$$
Z_{t}=X_{t}+\xi_{t}=\phi\left(F_{\alpha}^{t}\left(X_{0}, Y_{0}\right)\right)+\xi_{t}, \quad \text { for } \quad t \in \mathbf{Z},
$$


where $\left(X_{0}, Y_{0}\right) \in \mathbf{R}^{2}$ and $\left\{\xi_{t}\right\}_{t \in \mathbf{Z}}$ is a white noise process, will be given explicitly (see Theorem 1 in Section 3 ) by

$f_{Z}(\lambda)=f_{X}(\lambda)+\frac{\sigma_{\xi}^{2}}{2 \pi}=\frac{1}{2 \pi}\left[\gamma\left(e^{i \lambda}\right)+\gamma\left(e^{-i \lambda}\right)-\rho_{X}(0)\right]+\frac{\sigma_{\xi}^{2}}{2 \pi}$, for all $\lambda \in(-\pi, \pi]$,

where $\gamma$ is given by the last equality in (2.13) at Proposition 5 .

We will show the consistency of the periodogram in this situation. We shall also estimate the parameter $\alpha$ based on a time series.

Keywords: Spectral density; chaotic time series; dynamical system; periodogram.

\section{INTRODUCTION}

We shall present a complete spectral analysis of the stationary stochastic process

$Z_{t}=X_{t}+\xi_{t}=\phi\left(F_{\alpha}^{t}\left(x_{0}, y_{0}\right)\right)+\xi_{t}=\phi\left(F_{\alpha}\left(X_{t-1}, Y_{t-1}\right)+\xi_{t}, \quad\right.$ for $\quad t \in \mathbf{Z}$,

where $\phi(x, y)=x$ is a random variable, $\xi_{t}$ is a white noise process, and $F_{\alpha}$ is a transformation defined below.

The map $F_{\alpha}$ is defined from $K=([0,1] \times(0, \alpha)) \cup([0, \alpha] \times[\alpha, 1])$ to itself and it is given by $F_{\alpha}(x, y)=\left(T_{\alpha}(x), G_{\alpha}(x, y)\right)$ where the transformation $T_{\alpha}:[0,1] \rightarrow[0,1]$ is defined by

$$
T_{\alpha}(x)=\left\{\begin{array}{lll}
\frac{x}{\alpha}, & \text { if } & 0 \leq x<\alpha \\
\frac{\alpha(x-\alpha)}{1-\alpha}, & \text { if } & \alpha \leq x \leq 1,
\end{array}\right.
$$

with $\alpha \in(0,1)$ as a constant, and

$$
G_{\alpha}(x, y)= \begin{cases}\alpha y & , 0 \leq x<\alpha \\ \alpha+\left(\frac{1-\alpha}{\alpha}\right) y & , \quad \alpha \leq x \leq 1 .\end{cases}
$$

The graph of the map $T_{\alpha}$ is shown in Figure 1. The action of the difeomorphism $F_{\alpha}$ is presented in Figure 2. The transformation $F_{\alpha}$ is a modification of the well known Baker transformation. 
The map $T_{\alpha}$ describes a model for a particle that moves around in the interval $[0,1]$. If the particle is at position $x$, then after a unit of time it jumps to $T_{\alpha}(x)$ and so on. According to the model considered here suppose the spatial position of the particle is $T_{\alpha}^{t}(x)=X_{t}, t \in \mathrm{N}$, in the interval $[0,1]$. If the particle $X_{t}$ is in the interval $[0, \alpha)$, it has a uniformly spread possibility to jump to any point $X_{t+1}$ in $[0,1]$. However, if it is in the interval $[\alpha, 1)$ it has a uniformly spread possibility to jump to any point $X_{t+1}$ in the interval $[0, \alpha)$.

We are primarily interested in the map $T_{\alpha}$, but for defining the spectral density we need a bijective map. Therefore, we have to consider $F_{\alpha}$, the natural extension of $T_{\alpha}$ (Bogomolny and Carioli (1995)).

The difeomorphism $F_{\alpha}$ leaves invariant (see definition in Lopes and Lopes (1995)) an ergodic probability $\nu$ on $K \subset \mathbf{R}^{2}$, absolutely continuous with respect to the Lebesgue measure, that will be described in Section 3.

Choosing a point $\left(x_{0}, y_{0}\right)$ at random, according to the Lebesgue measure (or according to $\nu$ ), the spectral properties of the process $Z_{t}$ will be analyzed.

ore precisely, we shall present explicitly the analytic expression of the spectral density function of such stochastic process (see Section 3).

We refer the reader to Lopes and Lopes (1995) for general definitions and more detailed explanations for the context of the class of problems we consider here.

In Section 2, we present the basic results for a map $T_{\alpha}$ that are used in Section 3 for obtaining results for the map $F_{\alpha}$. In the appendix, we show the consistency of the periodogram in the model considered here.

The main result of this paper, the expression for the spectral density of $F_{\alpha}$, is presented in Theorem 1 in Section 3.

The explicit expression of the spectral density function (as obtained here) of a stochastic process allows one to analyze the efficiency of a given numerical method for estimating the spectrum, based on the closeness of the estimation obtained from the method compared to the true spectral density function.

We also estimate the parameter $\alpha$ at the end of Section 2 .

We refer the reader to Lopes and Lopes (1995) whenever definitions are used on this paper.

\section{THE AUTOCORRELATION FUNCTION}

Before considering the transformation $F_{\alpha}$ we will need to consider the 


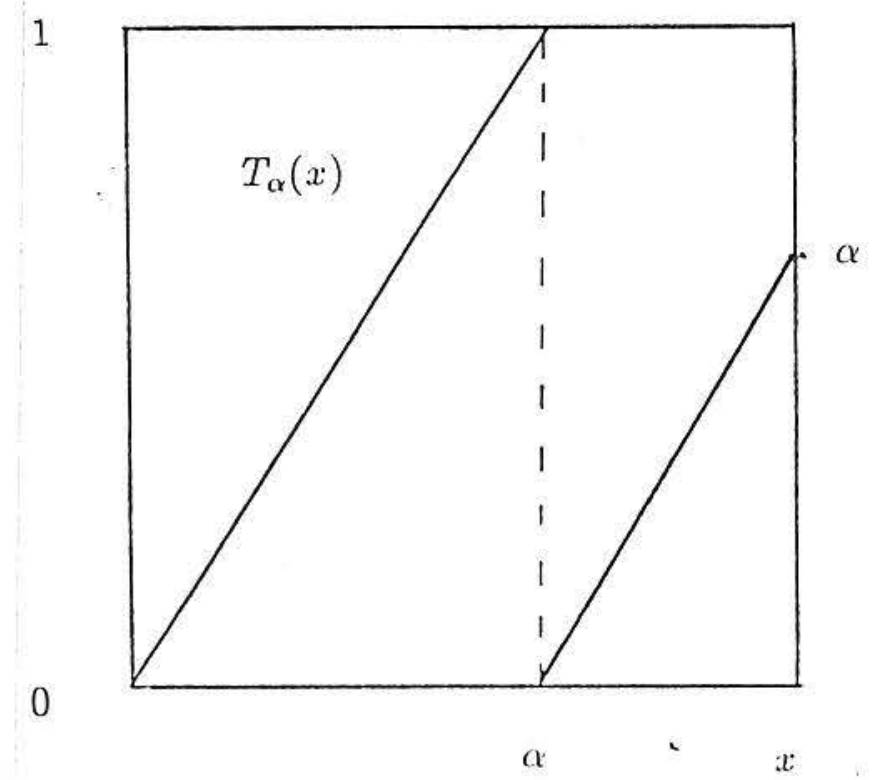

FIGURE 1 


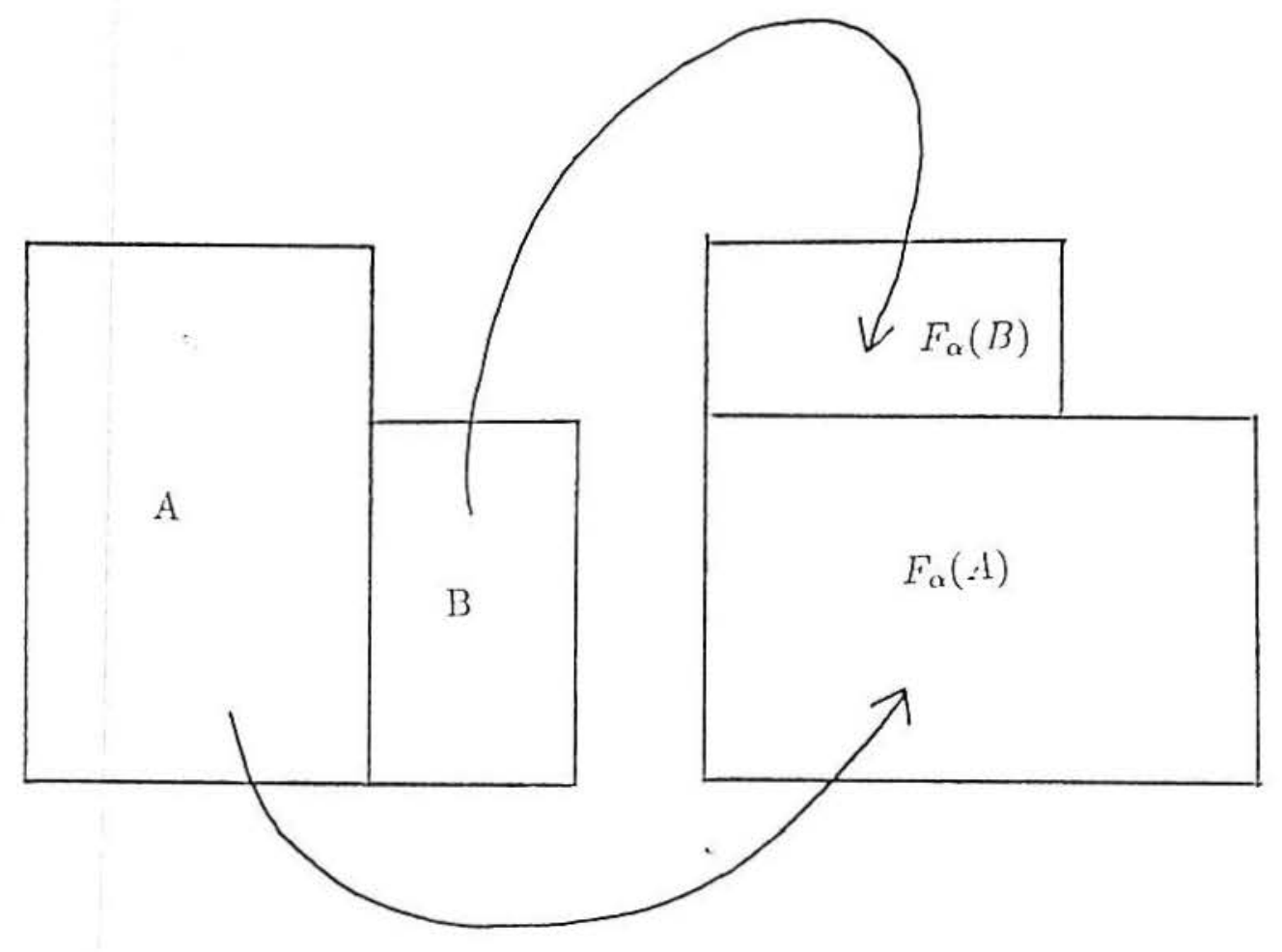

FIGURE 2 
transformation $T_{\alpha}$.

Let the transformation $T_{\alpha}:[0,1] \rightarrow[0,1]$ be given as in $(1.2)$, where $\alpha \in(0,1)$ is a constant. The derivative of $T_{\alpha}(x)$ at $x$ is $a=1 / \alpha$ if $0 \leq x<\alpha$ and $b=\alpha / 1-\alpha$ if $\alpha \leq x \leq 1$.

One observes that $\underline{a}$ is always greater than 1 , however $b \leq 1 \Leftrightarrow \alpha \leq 1 / 2$ and $b>1 \Leftrightarrow \alpha>1 / 2$. The transformation $T_{\alpha}$ is an expansive map (see Robinson (1995)) when $\alpha>1 / 2$. It is easy to show that when $\alpha<1 / 2, T_{\alpha}^{2}$ is an expansive map.

We will be interested here in finding the invariant measure $\mu$ absolutely continuous with respect to the Lebesgue measure (see Parry and Pollicott (1990); Robinson (1995); Ruelle (1978)) and also in analyzing the autocorrelation function associated with the stationary stochastic process $\left(T_{\alpha}^{t}, \mu\right)$.

First we shall find the invariant measure for the transformation $T_{\alpha}$. The transformation $T_{\alpha}$ has an invariant absolutely continuous measure $\mu$ with respect to the Lebesgue measure, if and only if, the Ruelle-Perron-Frobenius equation is satisfied (see Parry and Pollicott (1990); Ruelle (1978)), that is, if there exists a density function $g(x)$ such that

$$
g(y)=\sum_{x: g(x)=y} g(x) \frac{1}{T_{\alpha}^{\prime}(x)}
$$

and $d \mu=g(x) d x$. The above equation implies that if $0 \leq y<\alpha$ then

$$
g(y)=g\left(x_{1}\right) \frac{1}{T_{\alpha}^{\prime}\left(x_{1}\right)}+g\left(x_{2}\right) \frac{1}{T_{\alpha}^{\prime}\left(x_{2}\right)},
$$

for $0 \leq x_{1}<\alpha<x_{2} \leq 1$, and if $\alpha \leq y \leq 1$ then

$$
g(y)=g\left(x_{1}\right) \frac{1}{T_{\alpha}^{\prime}\left(x_{1}\right)}, \quad \text { for } 0 \leq x_{1}<\alpha .
$$

Therefore, the Ruelle-Perron-Frobenius equation in this situation is given by

$$
g(y)=\alpha g\left(x_{1}\right)+\left(\frac{1-\alpha}{\alpha}\right) g\left(x_{2}\right), \quad 0 \leq x_{1}<\alpha<x_{2} \leq 1 \text { and } 0 \leq y<\alpha
$$

and

$$
g(y)=\alpha g\left(x_{1}\right), \quad 0 \leq x_{1}<\alpha \text { and } \alpha \leq y \leq 1 .
$$


One considers the following density function

$g(x)=\left\{\begin{array}{lll}\frac{1}{\alpha(2-\alpha)}, & \text { if } & 0 \leq x<\alpha \\ \frac{1}{2-\alpha}, & \text { if } & \alpha \leq x \leq 1 .\end{array}\right.$

For the above guess of the density function, it is easy to see that equations in (2.2) hold.

Therefore, the Ruelle-Perron-Frobenius equations are satisfied and the density function $g(x)$ given in expression (2.3) defines an invariant measure $\mu$ such that, for any Borel set $A, \mu(A)=\int_{A} g(x) d x$.

Consider in the sequel the following notation

$c=\frac{1}{\alpha(2-\alpha)} \quad$ and $\quad d=\frac{1}{2-\alpha}$.

When $T$ is expansive the measure $\mu$ is an ergodic one (see Parry and Pollicott (1990)). Hence, the measure $\mu$ given by the expression (2.3) is an ergodic measure (applying the last statement for $T_{\alpha}$ or $T_{\alpha}^{2}$ ).

In an analogous way as in Lopes and Lopes (1995), consider $F_{\alpha}: K \rightarrow K$ ( $\mathrm{K}$ will be defined later), the natural extension of $T_{\alpha}$ (see Bogomolny and Carioli (1995)). We shall give the explicit expression for the spectral density function of the stationary stochastic process $F_{\alpha}^{t}=X_{t}$, the random variable $\phi(x, y)=x$ and a measure $\nu$ that will be defined later.

The reason to consider $F_{\alpha}(x, y)$ and not $T_{\alpha}(x)$ in our reasoning is because $F_{\alpha}$ is a bijective map while $T_{\alpha}$ is not.

Consider now the stationary stochastic process given by (1.1), where $\left\{\xi_{t}\right\}_{t \in \mathbf{Z}}$ is a noise process. For simplicity of the exposition we suppose $\xi_{t} \sim N\left(0, \sigma_{\xi}^{2}\right)$, for any $t \in \mathrm{Z}$, that is, a Gaussian white noise process. We assume that $\left\{\left(X_{t}, Y_{t}\right)\right\}_{t \in \mathbf{Z}}$ and $\left\{\xi_{t}\right\}_{t \in \mathbf{Z}}$ are uncorrelated processes.

Define the autocorrelation function of order $k$ of the process $\left\{X_{t}\right\}_{t \in \mathbf{Z}}$ by

$$
\begin{gathered}
\rho_{X}(k)=\frac{\operatorname{Cov}\left(X_{t}, X_{t+k}\right)}{\sqrt{\operatorname{Var}\left(X_{t}\right) \operatorname{Var}\left(X_{t+k}\right)}}= \\
=\frac{E\left[X_{t} \phi\left(F_{\alpha}^{k}\left(X_{t}, Y_{t}\right)\right)\right]-E\left(X_{t}\right) E\left[\phi\left(F_{\alpha}^{k}\left(X_{t}, Y_{t}\right)\right)\right]}{\sqrt{\operatorname{Var}\left(X_{t}\right) \operatorname{Var}\left[\phi\left(F_{\alpha}^{k}\left(X_{t}, Y_{t}\right)\right)\right]}}=
\end{gathered}
$$




$$
=\frac{E\left(x T_{\alpha}^{k}(x)\right)-E\left(x^{2}\right)}{\operatorname{Var}(x)} .
$$

Our goal is to derive the spectral density function of the process $\left\{X_{t}\right\}_{t \in \mathbf{Z}}$

$$
f_{X}(\lambda)=\frac{1}{2 \pi} \sum_{k=-\infty}^{\infty} \exp -i k \lambda \rho_{X}(k), \quad \text { for any } \quad \lambda \in(-\pi, \pi] .
$$

Hence, one needs to derive the autocorrelation function, $\rho_{X}(k)$, defined above.

By abuse of the notation, we shall denote $\phi(x)=x$ and $\phi(x, y)=x$ by the same letter $\phi$.

In an analogous way as in Lopes and Lopes (1995; Section 2), we will show that for positive $k$ the autocorrelation function of order $k$ of the dynamical systems $\left(F_{\alpha}(x, y), \phi(x, y), \nu\right)$ and $\left(T_{\alpha}(x), \phi(x), \mu\right)$ are the same. For negative values of $k$ the autocorrelation function of order $k$ of $F_{\alpha}$ is equal to the corresponding autocorrelation function of positive lag $k$ of $\left(T_{\alpha}, \phi, \mu\right)$. These properties will be described in Section 2.

There is no meaning for the autocorrelation function of $T_{\alpha}$ at negative lag $k$ because $T_{\alpha}$ is not an invertible map. $T_{\alpha}$.

First one needs three technical propositions involving the transformation

The following proposition gives a characterization of the $k$-th iterated of the transformation $T_{\alpha}(x)$ by a recursive formula.

PROPOSITION 1: The $k$-th iterated of the transformation $T_{\alpha}(x)$ given by the expression (1.2) is defined by

$$
T_{\alpha}^{k}(x)= \begin{cases}T_{\alpha}^{k-1}\left(\frac{x}{\alpha}\right), & \text { if } 0 \leq x<\alpha \\ T_{\alpha}^{k-2}\left(\frac{x-\alpha}{1-\alpha}\right), & \text { if } \quad \alpha \leq x \leq 1,\end{cases}
$$

for any integer $k \leq 2$.

PROOF: The proof is given by an induction in $k$. First one wants to show that the expression (2.7) holds for $k=2$ knowing that 


$$
T_{\alpha}^{2}(x)=\left\{\begin{array}{lll}
\frac{x}{\alpha^{2}}, & \text { if } & 0 \leq x<\alpha^{2} \\
\frac{x-\alpha^{2}}{1-\alpha}, & \text { if } & \alpha^{2} \leq x<\alpha \\
\frac{x-\alpha}{1-\alpha}, & \text { if } & \alpha \leq x \leq 1 .
\end{array}\right.
$$

Since $T_{\alpha}^{0} \equiv I d$, by using the recurrence formula when $k=2$ one has

$$
T_{\alpha}^{2}(x)=\left\{\begin{array}{lll}
T_{\alpha}^{1}\left(\frac{x}{\alpha}\right), & \text { if } & 0 \leq x<\alpha \\
\frac{x-\alpha}{1-\alpha}, & \text { if } & \alpha \leq x \leq 1 .
\end{array}\right.
$$

We know that

$$
T_{\alpha}^{1}\left(\frac{x}{\alpha}\right)=\frac{\frac{x}{\alpha}}{\alpha}=\frac{x}{\alpha^{2}}, \quad \text { if } \quad 0 \leq \frac{x}{\alpha}<\alpha
$$

that is,

$$
T_{\alpha}^{1}\left(\frac{x}{\alpha}\right)=\frac{x}{\alpha^{2}}, \quad \text { if } \quad 0 \leq x<\alpha^{2}
$$

or

$$
T_{\alpha}^{1}\left(\frac{x}{\alpha}\right)=\frac{\alpha\left(\frac{x}{\alpha}-\alpha\right)}{1-\alpha}=\frac{x-\alpha^{2}}{1-\alpha}, \quad \text { if } \quad \alpha \leq \frac{x}{\alpha} \leq 1
$$

that is,

$$
T_{\alpha}^{1}\left(\frac{x}{\alpha}\right)=\frac{x-\alpha^{2}}{1-\alpha}, \quad \text { if } \quad \alpha^{2} \leq x \leq \alpha
$$

Therefore, we have

$$
T_{\alpha}^{2}(x)=\left\{\begin{array}{lll}
\frac{x}{\alpha^{2}}, & \text { if } & 0 \leq x<\alpha^{2} \\
\frac{x-\alpha^{2}}{1-\alpha}, & \text { if } & \alpha^{2} \leq x<\alpha \\
\frac{x-\alpha}{1-\alpha}, & \text { if } \quad & \alpha \leq x \leq 1 .
\end{array}\right.
$$


Hence, the recurrence formula holds for $k=2$. Suppose now that (2.7) holds for $k$. One wants to show it also holds for $k+1$. Suppose $0 \leq x<\alpha$. Then,

$$
T_{\alpha}^{k+1}(x)=T_{\alpha}^{k}\left(T_{\alpha}(x)\right)=T_{\alpha}^{k}\left(\frac{x}{\alpha}\right) .
$$

If $\alpha \leq x \leq 1$ then

$$
\begin{aligned}
T_{\alpha}^{k+1}(x) & =T_{\alpha}^{k}\left(T_{\alpha}(x)\right)=T_{\alpha}^{k}\left(\frac{\alpha(x-\alpha)}{1-\alpha}\right)= \\
& =T_{\alpha}^{k-1}\left(T_{\alpha}\left(\frac{\alpha(x-\alpha)}{1-\alpha}\right)\right)=T_{\alpha}^{k-1}\left(\frac{x-\alpha}{1-\alpha}\right)
\end{aligned}
$$

since $\alpha(x-\alpha) /(1-\alpha) \leq \alpha$ whenever $x \in[0,1]$. Therefore,

$$
T_{\alpha}^{k+1}(x)=\left\{\begin{array}{lll}
T_{\alpha}^{k}\left(\frac{x}{\alpha}\right), & \text { if } & 0 \leq x<\alpha \\
T_{\alpha}^{k-1}\left(\frac{x-\alpha}{1-\alpha}\right), & \text { if } & \alpha \leq x \leq 1
\end{array}\right.
$$

and the proposition is proved.

PROPOSITION 2: The integral

$$
A(k)=\int_{0}^{1} T_{\alpha}^{k}(x) d x
$$

satisfies the recursive equation

$$
A(k)=\alpha A(k-1)+(1-\alpha) A(k-2),
$$

for any integer $k \geq 2$, with initial values $A(0)=1 / 2$ and $A(1)=$ $(2-\alpha) \alpha / 2$.

PROOF: From Proposition 1 one has, for any integer $k \geq 2$,

$$
A(k)=\int_{0}^{1} T_{\alpha}^{k}(x) d x=\int_{0}^{\alpha} T_{\alpha}^{k-1}\left(\frac{x}{\alpha}\right) d x+\int_{\alpha}^{1} T_{\alpha}^{k-2}\left(\frac{x-\alpha}{1-\alpha}\right) d x .
$$


By changing the variable $x$ to $y=x / \alpha$ in the first and the variable $x$ to $z=(x-\alpha) /(1-\alpha)$ in the second above integrals one has

$A(k)=\int_{0}^{1} T_{\alpha}^{k-1}(y) \alpha d y+\int_{0}^{1} T_{\alpha}^{k-2}(z)(1-\alpha) d z=\alpha A(k-1)+(1-\alpha) A(k-2)$,

for any integer $k \geq 2$. So, the equation (2.8) holds. Now one observes that

$$
A(0)=\int_{0}^{1} T_{\alpha}^{0}(x) d x=\int_{0}^{1} x d x=\frac{1}{2}
$$

and

$$
\begin{aligned}
A(1) & =\int_{0}^{1} T_{\alpha}^{1}(x) d x=\int_{0}^{\alpha} \frac{x}{\alpha} d x+\int_{\alpha}^{1} \alpha \frac{(x-\alpha)}{1-\alpha} d x= \\
& =\frac{\alpha}{2}+\frac{\alpha}{1-\alpha}\left(\frac{1}{2}-\alpha-\frac{\alpha^{2}}{2}+\alpha^{2}\right)= \\
& =\frac{\alpha}{2}+\frac{\alpha}{1-\alpha}\left(\frac{1}{2}-\alpha+\frac{\alpha^{2}}{2}\right)=\frac{\alpha}{2}+\frac{\alpha}{2}(1-\alpha)=\frac{\alpha}{2}(2-\alpha) .
\end{aligned}
$$

The proposition is proved.

PROPOSITION 3: The integral

$$
B(k)=\int_{0}^{1} x T_{\alpha}^{k}(x) d x
$$

satisfies the recursive equation

$$
B(k)=\alpha^{2} B(k-1)+(1-\alpha)^{2} B(k-2)+\alpha(1-\alpha) A(k-2),
$$

for any integer $k \geq 2$, with initial values $B(0)=1 / 3$ and $B(1)=$ $(1+\alpha)(2-\alpha) \alpha / 6$.

PROOF: From Proposition 1, for any integer $k \geq 2$,

$$
B(k)=\int_{0}^{1} x T_{\alpha}^{k}(x) d x=\int_{0}^{\alpha} x T_{\alpha}^{k-1}\left(\frac{x}{\alpha}\right) d x+\int_{\alpha}^{1} x T_{\alpha}^{k-2}\left(\frac{x-\alpha}{1-\alpha}\right) d x .
$$


By changing the variable $x$ to $y=x / \alpha$ in the first and the variable $x$ to $z=(x-\alpha) /(1-\alpha)$ in the second above integrals one has

$$
\begin{aligned}
B(k) & =\int_{0}^{1} \alpha y T_{\alpha}^{k-1}(y) \alpha d y+\int_{0}^{1}[z(1-\alpha)+\alpha] T_{\alpha}^{k-2}(z)(1-\alpha) d z= \\
& =\alpha^{2} \int_{0}^{1} y T_{\alpha}^{k-1}(y) d y+(1-\alpha)^{2} \int_{0}^{1} z T_{\alpha}^{k-2}(z) d z+ \\
& +\alpha(1-\alpha) \int_{0}^{1} T_{\alpha}^{k-2}(z) d z=\alpha^{2} B(k-1)+(1-\alpha)^{2} B(k-2)+ \\
& +\alpha(1-\alpha) A(k-2)
\end{aligned}
$$

for any integer $k \geq 2$. So, the equation (2.9) holds. The initial values are given by

$$
B(0)=\int_{0}^{1} x T_{\alpha}^{0}(x) d x=\int_{0}^{1} x^{2} d x=\frac{1}{3}
$$

and

$$
\begin{aligned}
B(1) & =\int_{0}^{1} x T_{\alpha}^{1}(x) d x=\int_{0}^{\alpha} x \frac{x}{\alpha} d x+\int_{\alpha}^{1} x \frac{\alpha(x-\alpha)}{1-\alpha} d x= \\
& =\frac{\alpha^{2}}{3}+\frac{\alpha}{1-\alpha}\left(\frac{1}{3}-\frac{\alpha}{2}-\frac{\alpha^{3}}{3}+\frac{\alpha^{3}}{2}\right)= \\
& =\frac{\alpha^{2}}{3}+\frac{\alpha}{6(1-\alpha)}\left(2-3 \alpha+\alpha^{3}\right)= \\
& =\frac{\alpha^{2}}{3}-\frac{\alpha}{6}\left(\alpha^{2}+\alpha-2\right)=\frac{\alpha}{6}\left(2+\alpha-\alpha^{2}\right)=\frac{\alpha}{6}(1+\alpha)(2-\alpha) .
\end{aligned}
$$

And the proposition is proved.

From Propositions 2 and 3 we shall derive the autocorrelation function $\rho_{X}(k), k \geq 0$, of the process $\left\{X_{t}\right\}_{t \in \mathrm{N}}$.

PROPOSITION 4: Let $\left\{X_{t}\right\}_{t \in \mathrm{N}}$ be the stationary stochastic process given in (2.5). The autocorrelation function of order $k$ of the process $\left\{X_{t}\right\}_{t \in \mathrm{N}}$ defined in expression (2.6) is given by

$\rho_{X}(k)=\frac{E\left[X_{t} T_{\alpha}^{k}\left(X_{t}\right)\right]-\left(\frac{1+\alpha-\alpha^{2}}{2(2-\alpha)}\right)^{2}}{\frac{\left(\alpha^{2}-\alpha+1\right)\left(\alpha^{2}-5 \alpha+5\right)}{12(2-\alpha)^{2}}}$, 
where $E\left[X_{t} T_{\alpha}^{k}\left(X_{t}\right)\right]$, denoted by $C(k)$, is given by the three term relation $C(k)=\alpha^{2} c B(k-1)+(1-\alpha)^{2} d B(k-2)+\alpha(1-\alpha) d A(k-2)$,

for any integer $k \geq 2$, with $A(k)$ given by (2.8), $B(k)$ given by (2.9) and the constants $c$ and $d$ are defined in the expression (2.4). Moreover, the initial values $C(0)$ and $C(1)$ are given by

$$
C(0)=\frac{1+\alpha^{2}-\alpha^{3}}{3(2-\alpha)} \quad \text { and } \quad C(1)=\frac{\alpha\left(4-\alpha-\alpha^{2}\right)}{6(2-\alpha)}
$$

PROOF: From the stationarity of the process $\left\{X_{t}\right\}_{t \in \mathrm{N}}$, one observes that

$$
E\left(X_{t}\right)=E\left(X_{t+k}\right) \equiv E\left(T_{\alpha}^{k}\left(X_{t}\right)\right)
$$

and

$$
\operatorname{Var}\left(X_{t}\right)=\operatorname{Var}\left(X_{t+k}\right) \equiv \operatorname{Var}\left(T_{\alpha}^{k}\left(X_{t}\right)\right) .
$$

The expected value of the process $\left\{X_{t}\right\}_{t \in \mathbb{N}}$ is given by

$$
\begin{aligned}
E\left(X_{t}\right) & =\int_{0}^{1} x d \mu(x)=c \int_{0}^{\alpha} x d x+d \int_{\alpha}^{1} x d x= \\
& =\frac{\alpha^{2}}{2 \alpha(2-\alpha)}+\frac{1-\alpha^{2}}{2(2-\alpha)}=\frac{1+\alpha-\alpha^{2}}{2(2-\alpha)} .
\end{aligned}
$$

The second moment of the process $\left\{X_{t}\right\}_{t \in \mathrm{N}}$ is given by

$$
\begin{aligned}
E\left(X_{t}^{2}\right) & =\int_{0}^{1} x^{2} d \mu(x)=c \int_{0}^{\alpha} x^{2} d x+d \int_{\alpha}^{1} x^{2} d x= \\
& =\frac{\alpha^{2}}{3(2-\alpha)}+\frac{1-\alpha^{3}}{3(2-\alpha)}=\frac{1+\alpha^{2}-\alpha^{3}}{3(2-\alpha)} .
\end{aligned}
$$

Hence, the variance of the process $\left\{X_{t}\right\}_{t \in \mathrm{N}}$ is given by

$$
\begin{aligned}
\operatorname{Var}\left(X_{t}\right) & =E\left(X_{t}^{2}\right)-\left[E\left(X_{t}\right)\right]^{2}=\frac{1+\alpha^{2}-\alpha^{3}}{3(2-\alpha)}-\left(\frac{1+\alpha-\alpha^{2}}{2(2-\alpha)}\right)^{2}= \\
& =\frac{\alpha^{4}-6 \alpha^{3}+11 \alpha^{2}-10 \alpha+5}{12(2-\alpha)^{2}}=\frac{\left(\alpha^{2}-\alpha+1\right)\left(\alpha^{2}-5 \alpha+5\right)}{12(2-\alpha)^{2}} .
\end{aligned}
$$


The autocorrelation function of order $k$ of the process $\left\{X_{t}\right\}_{t \in \mathrm{N}}$ is defined in (2.6) where $E\left[X_{t} T_{\alpha}^{k}\left(X_{t}\right)\right]$ is given by

$$
\begin{aligned}
C(k) & \equiv E\left[X_{t} T_{\alpha}^{k}\left(X_{t}\right)\right]=\int_{0}^{1} x T_{\alpha}^{k}(x) d \mu(x)= \\
& =c \int_{0}^{\alpha} x T_{\alpha}^{k}(x) d x+d \int_{\alpha}^{1} x T_{\alpha}^{k}(x) d x= \\
& =c \int_{0}^{\alpha} x T_{\alpha}^{k-1}\left(\frac{x}{\alpha}\right) d x+d \int_{\alpha}^{1} x T_{\alpha}^{k-2}\left(\frac{x-\alpha}{1-\alpha}\right) d x .
\end{aligned}
$$

The last above equality is due to Proposition 1 . By changing the variable $x$ to $y=x / \alpha$ in the first and variable $x$ to $z=(x-\alpha) /(1-\alpha)$ in the second above integrals one has

$$
\begin{aligned}
C(k) & =c \int_{0}^{1} \alpha y T_{\alpha}^{k-1}(y) \alpha d y+d \int_{0}^{1}((1-\alpha) z+\alpha) T_{\alpha}^{k-2}(z)(1-\alpha) d z= \\
& =c \alpha^{2} \int_{0}^{1} y T_{\alpha}^{k-1}(y) d y+d\left(1-\alpha^{2}\right) \int_{0}^{1} z T_{\alpha}^{k-2}(z) d z+ \\
& +d \alpha(1-\alpha) \int_{0}^{1} T_{\alpha}^{k-2}(z) d z= \\
& =\alpha^{2} c B(k-1)+(1-\alpha)^{2} d B(k-2)+\alpha(1-\alpha) d A(k-2),
\end{aligned}
$$

for any integer $k \geq 2$, where $A(k)$ is given by $(2.8), B(k)$ is given by (2.9) and the constants $c$ and $d$ by (2.4). Hence, the expression (2.11) holds.

One observes that the initial values for $C(k)$ are given by

$$
\begin{aligned}
C(0) & =\int_{0}^{1} x T_{\alpha}^{0}(x) d \mu(x)=\int_{0}^{1} x^{2} d \mu(x)= \\
& =c \int_{0}^{\alpha} x^{2} d x+d \int_{\alpha}^{1} x^{2} d x=c \frac{\alpha^{3}}{3}+\frac{d}{3}\left(1-\alpha^{3}\right)=\frac{1+\alpha^{2}-\alpha^{3}}{3(2-\alpha)}
\end{aligned}
$$

and

$$
\begin{aligned}
C(1) & =\int_{0}^{1} x T_{\alpha}^{1}(x) d \mu(x)=c \int_{0}^{\alpha} x T_{\alpha}(x) d x+d \int_{\alpha}^{1} x T_{\alpha}(x) d x= \\
& =c \int_{0}^{\alpha} x \frac{x}{\alpha} d x+d \int_{\alpha}^{1} x \alpha \frac{(x-\alpha)}{1-\alpha} d x= \\
& =\frac{2 \alpha-\alpha^{3}-\alpha^{2}+2 \alpha}{6(2-\alpha)}=\frac{\alpha\left(4-\alpha-\alpha^{2}\right)}{6(2-\alpha)},
\end{aligned}
$$


and the Proposition is proved.

From (2.10) we still need to know the quantity $C(k)$ in order to give the autocorrelation function $\rho_{X}(k)$ of the process $\left\{X_{t}\right\}_{t \in \mathbf{Z}}$. One can equivalently describe the quantities $A(k), B(k)$ and $C(k)$ by the following power series $\varphi(z)=\sum_{k \geq 0} A(k) z^{k}, \Psi(z)=\sum_{k \geq 0} B(k) z^{k} \quad$ and $\quad \gamma(z)=\sum_{k \geq 0} C(k) z^{k}$.

PROPOSITION 5: The power series for $A(k), B(k)$ and $C(k)$ as in expression (2.12) are given, respectively, by

$$
\begin{aligned}
\varphi(z) & =\frac{1+\alpha z(1-\alpha)}{2[(1-\alpha) z+1](1-z)}, \\
\psi(z) & =\frac{2-\alpha z\left(\alpha^{2}+\alpha-2\right)+6 \alpha(1-\alpha) z^{2} \varphi(z)}{6\left[1-\alpha^{2} z-(1-\alpha)^{2} z^{2}\right]} \text { and } \\
\gamma(z) & =\frac{2 \alpha^{2}(1-\alpha)+2+\alpha z\left(2-\alpha-\alpha^{2}\right)}{6(2-\alpha)}+ \\
& +\left[\frac{\alpha z+(1-\alpha)^{2} z^{2}}{2-\alpha}\right] \psi(z)+\frac{\alpha(1-\alpha) z^{2}}{2-\alpha} \varphi(z) .
\end{aligned}
$$

PROOF: From Proposition 2 one has the recursive formula for $A(k)$, for any integer $k \geq 2$, and the two initial values. Hence,

$$
\begin{aligned}
\varphi(z) & =A(0)+A(1) z+\sum_{k \geq 2}[\alpha A(k-1)+(1-\alpha) A(k-2)] z^{k}= \\
& =A(0)+A(1) z+\alpha \sum_{k \geq 2} A(k-1) z^{k}+(1-\alpha) \sum_{k \geq 2} A(k-2) z^{k}= \\
& =\frac{1}{2}+\frac{\alpha}{2}(2-\alpha) z+\alpha z[\varphi(z)-A(0)]+(1-\alpha) z^{2} \varphi(z)= \\
& =\frac{1}{2}+\frac{\alpha}{2} z(2-\alpha)+\alpha z \varphi(z)-\frac{\alpha z}{2}+(1-\alpha) z^{2} \varphi(z) .
\end{aligned}
$$

Therefore,

$$
\varphi(z)=\frac{1+\alpha z-\alpha^{2} z}{2\left[1-\alpha z-(1-\alpha) z^{2}\right]}=\frac{1+\alpha z(1-\alpha)}{2[(1-\alpha) z+1](1-z)} .
$$


And the first equality in (2.13) holds.

To prove the second equality in (2.13) one considers the recursive formula for $B(k)$, for any integer $k \geq 2$, with two initial values given in Proposition 3. Hence,

$$
\begin{aligned}
\psi(z) & =\sum_{k \geq 0} B(k) z^{k}=B(0)+B(1) z+\sum_{k \geq 2}\left[\alpha^{2} B(k-1)+\right. \\
& \left.+(1-\alpha)^{2} B(k-2)+\alpha(1-\alpha) A(k-2)\right] z^{k}=B(0)+B(1) z+ \\
& +\alpha^{2} \sum_{k \geq 2} B(k-1) z^{k}+(1-\alpha)^{2} \sum_{k \geq 2} B(k-2) z^{k}+ \\
& +\alpha(1-\alpha) \sum_{k \geq 2} A(k-2) z^{k}=\frac{1}{3}+\frac{\alpha}{6} z(1+\alpha)(2-\alpha)+ \\
& +\alpha^{2} z \psi(z)-\frac{\alpha^{2} z}{3}+(1-\alpha)^{2} z^{2} \psi(z)+\alpha(1-\alpha) z^{2} \varphi(z) .
\end{aligned}
$$

Therefore,

$$
\begin{aligned}
\psi(z) & =\frac{2+\alpha(1+\alpha)(2-\alpha) z-2 \alpha^{2} z+6 \alpha(1-\alpha) z^{2} \varphi(z)}{6\left[1-\alpha^{2} z-(1-\alpha)^{2} z^{2}\right]}= \\
& =\frac{2-\alpha z\left(\alpha^{2}+\alpha-2\right)+6 \alpha(1-\alpha) z^{2} \varphi(z)}{6\left[1-\alpha^{2} z-(1-\alpha)^{2} z^{2}\right]} .
\end{aligned}
$$

And the second equality in (2.13) holds. To prove the third equality in (2.13) one considers the recursive formula for $C(k)$, for any integer $k \geq 2$, with two initial values given in Proposition 4. Hence,

$$
\begin{aligned}
\gamma(z) & =\sum_{k \geq 0} C(k) z^{k}=C(0)+C(1) z+\sum_{k \geq 2} \alpha^{2} c B(k-1) z^{k}+ \\
& +\sum_{k \geq 2}(1-\alpha)^{2} d B(k-2)+\alpha(1-\alpha) d A(k-2) z^{k}= \\
& =C(0)+C(1) z+\alpha^{2} c \sum_{k \geq 2} B(k-1) z^{k}+(1-\alpha)^{2} d \sum_{k \geq 2} B(k-2) z^{k}+ \\
& +\alpha(1-\alpha) d \sum_{k \geq 2} A(k-2) z^{k}= \\
& =\frac{1+\alpha^{2}-\alpha^{3}}{3(2-\alpha)}+\frac{\alpha\left(4-\alpha-\alpha^{2}\right) z}{6(2-\alpha)}+\alpha^{2} c z[\psi(z)-B(0)]+
\end{aligned}
$$




$$
\begin{aligned}
& +(1-\alpha)^{2} d z^{2} \psi(z)+\alpha(1-\alpha) d z^{2} \varphi(z)= \\
& =\frac{2\left(1+\alpha^{2}-\alpha^{3}\right)+\alpha\left(4-\alpha-\alpha^{2}\right) z}{6(2-\alpha)}+\alpha^{2} c z \psi(z)-\frac{1}{3} \alpha^{2} c z+ \\
& +(1-\alpha)^{2} d z^{2} \psi(z)+\alpha(1-\alpha) d z^{2} \varphi(z)= \\
& =\frac{2\left(1+\alpha^{2}-\alpha^{3}\right)+\alpha z\left(2-\alpha-\alpha^{2}\right)}{6(2-\alpha)}+\left[\frac{\alpha z+(1-\alpha)^{2} z^{2}}{2-\alpha}\right] \psi(z)+ \\
& +\frac{\alpha(1-\alpha) z^{2}}{2-\alpha} \varphi(z)
\end{aligned}
$$

Therefore,

$$
\begin{aligned}
\gamma(z) & =\frac{2 \alpha^{2}(1-\alpha)+2+\alpha z\left(2-\alpha-\alpha^{2}\right)}{6(2-\alpha)}+ \\
& +\frac{1}{2-\alpha}\left[\left(\alpha z+(1-\alpha)^{2} z^{2}\right) \psi(z)+\alpha(1-\alpha) z^{2} \varphi(z)\right]
\end{aligned}
$$

Hence, the proposition is proved.

Note that the estimation of $\alpha$ for the stationary stochastic process (1.1) can be obtained from Proposition 4. This follows from the fact that

$$
\frac{\alpha\left(4-\alpha-\alpha^{2}\right)}{6(2-\alpha)}=C(1)=\int_{0}^{1} x T_{\alpha}^{0}(x) d \mu(x) .
$$

Considering a times series $\left\{Z_{t}\right\}_{t=1}^{N}$ and using the Birkhoff's Ergodic Theorem one can estimate $\alpha$ by solving the equation

$$
C \hat{(1)}=\frac{1}{N} \sum_{t=1}^{N-1} Z_{t} Z_{t+1}=\frac{\hat{\alpha}\left(4-\hat{\alpha}-\hat{\alpha}^{2}\right)}{6(2-\hat{\alpha})},
$$

in the variable $\hat{\alpha}$. 


\section{THE SPECTRAL DENSITY FUNCTION}

In this section we shall use the results for $T_{\alpha}$ obtained in the previous section for the map $F_{\alpha}$.

Now we define the natural extension $F_{\alpha}$ of the transformation $T_{\alpha}$. Consider the transformation $F_{\alpha}: K \rightarrow K$, where $K=([0,1] \times(0, \alpha)) \cup([0, \alpha] \times$ $[\alpha, 1])$, given by $F_{\alpha}(x, y)=\left(T_{\alpha}(x), G_{\alpha}(x, y)\right)$ where $G_{\alpha}(x, y)$ is defined by (1.2).

One observes that $F_{\alpha}$ is a homeomorphism of $K$ and $F_{\alpha}^{n}$ is of the form

$$
F_{\alpha}^{n}(x, y)=\left(T_{\alpha}^{n}(x), G_{\alpha, n}(x, y)\right),
$$

that is, the action of $F_{\alpha}$ in the first variable is just the action of $T_{\alpha}$.

Now we shall define the $F_{\alpha}$-invariant measure $\nu$ on $K$, absolutely continuous with respect to the Lebesgue measure $d x d y$.

For sets of the form $A_{1} \times A_{2}$, where $A_{1} \subset(0, \alpha)$ and $A_{2} \subset(\alpha, 1)$ or $A_{1} \subset(\alpha, 1)$ and $A_{2} \subset(0, \alpha)$, we define $\nu\left(A_{1} \times A_{2}\right)=(2-\alpha) \mu\left(A_{1}\right) \mu\left(A_{2}\right)$.

For sets of the form $A_{1} \times A_{2}$, where $A_{1} \subset(0, \alpha)$ and $A_{2} \subset(0, \alpha)$, we define $\nu\left(A_{1} \times A_{2}\right)=(2-\alpha) \alpha \mu\left(A_{1}\right) \mu\left(A_{2}\right)$.

It is not difficult to see that $\nu$ is invariant for $F_{\alpha}$ and is absolutely continuous with respect to the Lebesgue measure. The measure $\nu$ satisfies $\nu(A \times(0,1))=\mu(A)$, when $A \subset(0, \alpha)$ and $\nu(A \times(0, \alpha))=\mu(A)$, when $A \subset(\alpha, 1)$.

THEOREM 1: The spectral density function of the process

$$
Z_{t}=X_{t}+\xi_{t}=\phi\left(F_{\alpha}^{t}\left(X_{0}, Y_{0}\right)\right)+\xi_{t}, \quad \text { for } t \in \mathbf{Z}
$$

is given by

$$
f_{Z}(\lambda)=\frac{1}{2 \pi}\left[\gamma\left(e^{i \lambda}\right)+\gamma\left(e^{-i \lambda}\right)-\rho_{X}(0)\right]+\frac{\sigma_{\xi}^{2}}{2 \pi},
$$

for any $\lambda \in(-\pi, \pi]$, where $\gamma(z)$ is given by the third equality in expression (2.13) of Proposition 5. The point $\left(X_{0}, Y_{0}\right)$ is chosen randomly according to the measure $\nu$ (or according to the Lebesgue measure). 
PROOF: The integral of $\nu$ with respect to any function $H$ that depends only on the $x$ variable is such that

$$
\int H(x) d \nu(x, y)=\int H(x) d \mu(x) .
$$

One observes that $\phi(x, y)=x$ is a random variable and $F_{\alpha}: K \rightarrow K$ defines a stationary stochastic process $X_{t}=\phi\left(F_{\alpha}^{t}\left(X_{0}, Y_{0}\right)\right)$ with respect to the invariant probability $\nu$ defined above.

From the expression (2.15) and for any positive $t \in \mathbf{N}$,

$$
\int \phi\left(F_{\alpha}^{t}(x, y)\right) \phi(x, y) d \nu(x, y)=\int \phi\left(T_{\alpha}^{t}(x)\right) \phi(x) d \mu(x) .
$$

For any positive $t \in \mathbf{N}$ (that is, when $-t$ is negative)

$$
\int \phi\left(F_{\alpha}^{-t}(x, y)\right) \phi(x, y) d \nu(x, y)=\int \phi(x, y) \phi\left(F_{\alpha}^{t}(x, y)\right) d \nu(x, y)
$$

because $\nu$ is invariant for $F_{\alpha}$. Therefore, from (2.15) and for any positivé $t \in \mathbf{N}$

$$
\int \phi\left(F_{\alpha}^{-t}(x, y)\right) \phi(x, y) d \nu(x, y)=\int \phi\left(T_{\alpha}^{t}(x)\right) \phi(x) d \mu(x) .
$$

The conclusion is that the autocorrelation coefficients $C(t)=C(-t)$, $t \in \mathbf{N}$ of the stochastic process given by the random variable $\phi(x, y)=x$, the transformation $F_{\alpha}$ and the probability $\nu$ can be obtained from the autocorrelation coefficients obtained previously for the stochastic process given by the random variable $\phi(x)=x$, the transformation $T_{\alpha}$ and the probability $\mu$.

The spectral density function of the process $\left\{X_{t}\right\}_{t \in \mathrm{Z}}$ is given by

$$
f_{X}(\lambda)=\frac{1}{2 \pi} \sum_{k=-\infty}^{\infty} \exp (-i k \lambda) \rho_{X}(k) \geq 0,
$$

for all $\lambda \in(-\pi, \pi]$ (see Brockwell and Davis (1987)). Therefore, the spectral density function of the process (1.1) is (see (2.16)

$f_{Z}(\lambda)=f_{X}(\lambda)+\frac{\sigma_{\xi}^{2}}{2 \pi}=\frac{1}{2 \pi}\left[\gamma\left(e^{i \lambda}\right)+\gamma\left(e^{-i \lambda}\right)-\rho_{X}(0)\right]+\frac{\sigma_{\xi}^{2}}{2 \pi}$, for all $\lambda \in(-\pi, \pi]$, 
where $\gamma$ is explicitly given by the expression (2.13) in Proposition 5 .

From Parry and Pollicott (1990) it is known that $\rho_{X}(k)$ decays exponentially to zero, hence $f_{X}(\lambda)$ is an analytic function for any $\lambda \in(-\pi, \pi]$.

We can also analyze alternatively $f_{Z}(u), u \in(-1,1)$ when $u=\frac{\lambda}{\pi}$. We shall use this notation in the appendix. If $\rho_{X}(k)=\rho_{X}(-k)$ (as in the present case), one just needs to consider $f_{Z}(u), u \in(0,1)$, because $f_{Z}(u)=f_{Z}(-u)$.

\section{APPENDIX. THE CONSISTENCY OF THE PERIODOGRAM}

We analyze in this section the periodogram for $\left(\phi, T_{\alpha}\right)$ (or for $\left(\phi, F_{\alpha}\right)$ ). Our purpose here is to show how to obtain an approximation of the spectral measure $f_{X}(u)$ from a time series data $X_{t}=T_{\alpha}^{t}\left(X_{0}\right)$, for $t \in\{1, \cdots, N\}$, where $X_{0}$ is chosen at random according to the measure $\mu$ (or according to the Lebesgue measure). We can alternatively estimate

$$
\sum_{h=-\infty}^{\infty} E\left(X_{0} X_{h}\right) \exp (-2 \pi i h u)
$$

with $X_{h}=\phi\left(F_{\alpha}^{h}(x, y)\right)$ and from this result estimate the spectral measure $f_{X}(u)$. By abuse of the notation we shall also call the above expression as the spectral measure.

Note that as the random variable $\phi(x, y)$ depends only on $x$ (for positive $t, \phi\left(F_{\alpha}^{t}(x, y)\right)=T_{\alpha}^{t}(x)$ independently of $y$ ) we shall consider the periodogram for $T_{\alpha}$ instead of $F_{\alpha}$.

In fact, the proof presented here works for any expansive map $T$, any Holder random variable $\phi$ and the ergodic absolutely continuous invariant probability $\mu$ for $T$. We leave to the reader the extension of the reasoning below to such case.

Consider the transformation $T_{\alpha}:[0,1] \rightarrow[0,1]$, where $\alpha \in(0,1)$, given by (1.2). The map $T_{\alpha}$ (or $T_{\alpha}^{2}$ ) is an expanding one.

We shall assume, for the sake of simplicity, that $\phi$ is the random variable $\phi(x)=x$ and $d \mu(x)=g(x) d x$ is the unique ergodic and absolutely continuous invariant probability for $T_{\alpha}$.

The goal here is to sketch the proof of the smoothed periodogram's consistency in the above case. One denotes $X_{t}=\left(\phi \circ T_{\alpha}^{t}\right)\left(X_{0}\right)=T_{\alpha}^{t}\left(X_{0}\right)=$ $\phi\left(F_{\alpha}^{t}\left(X_{0}, Y_{0}\right)\right)$, and $\left\{X_{t}\right\}_{t=1}^{N}$ a time series of $N$ observations where $X_{0}$ is an initial point chosen randomly according to $\mu$. From the Birkhoff's Ergodic 
Theorem ( $\mu$ is ergodic for $T_{\alpha}$ ), for each subinterval $\Delta_{j}=\left(a_{j}, b_{j}\right) \subset[0,1]$ and for $\mu$-almost every $x_{0} \in[0,1]$

$$
\mu\left(\Delta_{j}\right)=\int_{\Delta_{j}} g(x) d x=\lim _{N \rightarrow \infty} \frac{1}{N}\left(\#\left\{t \mid 1 \leq t \leq N, T_{\alpha}^{t}\left(x_{0}\right) \in \Delta_{j}\right\}\right) .
$$

If $\left|b_{j}-a_{j}\right|=\epsilon$ is small and $N$ is large enough, then

$A_{N}(\epsilon)=\frac{1}{N}\left(\#\left\{t \mid 1 \leq t \leq N, T_{\alpha}^{t}\left(X_{0}\right) \in \Delta_{j}\right\}\right) \approx g\left(c_{j}\right) \Delta_{j}=B_{N}(\epsilon)$,

for some $c_{j}=c_{j}(N) \in \Delta_{j}$.

The expression $A_{N}(\epsilon) \approx B_{N}(\epsilon)$ means that the quotient $A_{N}(\epsilon) / B_{N}(\epsilon)$ goes to one when $N$ goes to infinity and $\epsilon$ goes to zero.

Consider the discrete Fourier transform of the spatial position of the data obtained as the sampled time series $X_{t}=T_{\alpha}^{t}\left(X_{0}\right)$, for $1 \leq t \leq N$,

$$
f(k)=\frac{1}{\sqrt{N}} \sum_{t=1}^{N} X_{t} \exp \left(-i \omega_{k} t\right)
$$

where $\omega_{k}=2 \pi k N^{-1}, k=1,2, \cdots, N$, are the so-called the Fourier frequencies of the time series $X_{t}, 1 \leq t \leq N$. The periodogram value $I\left(\omega_{k}\right)$ at the frequency $\omega_{k}$, for

$$
k \in\left\{j \in \mathrm{Z} ; 0<\omega_{j}=\frac{2 \pi j}{N} \leq 2 \pi\right\},
$$

is defined in terms of the discrete Fourier transform $f(k)$ of a sample $X_{t}$, for $1 \leq t \leq N$, by

$$
\begin{aligned}
I\left(\omega_{k}\right) & =f(k) \overline{f(k)}=\frac{1}{N} \sum_{t=1}^{N} X_{t} \exp \left(-i \omega_{k} t\right) \sum_{s=1}^{N} X_{s} \exp \left(i \omega_{k} s\right)= \\
& =\frac{1}{N} \sum_{t=1}^{N} \sum_{s=1}^{N} X_{t} X_{s} \exp \left(-i(t-s) \omega_{k}\right),
\end{aligned}
$$

where $\bar{z}$ denotes the complex conjugate of $z$.

For each $h \in \mathbf{Z}$ consider $t$ and $s$ such that $t-s=h$. Then,

$$
I\left(\omega_{k}\right)=\frac{1}{N}\left(\sum_{h=0}^{N-1} \sum_{s=1}^{N-h} X_{s} X_{s+h} \exp \left(-i h \omega_{k}\right)+\sum_{h=-1}^{1-N} \sum_{s=-h}^{N} X_{s} X_{s+h} \exp \left(-i h \omega_{k}\right)\right)=
$$




$$
\begin{array}{r}
=\frac{1}{N} \sum_{h=0}^{N-1} \sum_{s=1}^{N-h} X_{s} \phi\left(F_{\alpha}^{h}\left(X_{s}, Y_{s}\right)\right) \exp \left(-i h \omega_{k}\right)+ \\
+\frac{1}{N} \sum_{h=-1}^{1-N} \sum_{s=-h}^{N} X_{s} \phi\left(F_{\alpha}^{h}\left(X_{s}, Y_{s}\right)\right) \exp \left(-i h \omega_{k}\right) .
\end{array}
$$

Now if we take $\Delta_{j}, 1 \leq j \leq v$, as a partition by intervals (of the same size) of the interval $[0,1]$, with $\left|\Delta_{j}\right|=\epsilon=1 / v$ small, one observes from (2.17) that

$$
\frac{\#\left[X_{j} \in \Delta_{j}\right]}{N} \approx \Delta_{j} g\left(c_{j}\right)
$$

where $c_{j} \in \Delta_{j}, 1 \leq j \leq v$.

We shall sum up $X_{s}=T_{\alpha}^{s}\left(X_{0}\right)=\phi\left(F_{\alpha}^{s}\left(X_{0}, Y_{0}\right)\right)$ according to its position in each $\Delta_{j}$. Hence,

$$
\Delta_{j} g\left(c_{j}\right) N \approx \#\left\{s \mid 1 \leq s \leq N, X_{s} \in \Delta_{j}\right\} .
$$

Then, from (2.18)

$$
\begin{gathered}
I\left(\omega_{k}\right) \approx \frac{1}{N} \sum_{|h|<N} \sum_{j=1}^{v} c_{j} \phi\left(F_{\alpha}^{h}\left(c_{j}, y_{j}\right)\right)\left(\Delta_{j} g\left(c_{j}\right) N\right) \exp \left(-i h \omega_{k}\right)= \\
=\sum_{|h|<N} \sum_{j=1}^{v} c_{j} \phi\left(F_{\alpha}^{h}\left(c_{j}, y_{j}\right)\right) g\left(c_{j}\right) \Delta_{j} \exp \left(-i h \omega_{k}\right) .
\end{gathered}
$$

We shall show that for any $X_{0}$ chosen at random, then $\sum_{k=1}^{N} I\left(\omega_{k}\right) \delta_{\omega_{k}}$ converges in the distribution sense to the spectral density function

$$
\sum_{h \in \mathbf{Z}} E\left(X_{0} X_{h}\right) \exp (-2 \pi i h u)
$$

, where $\delta_{\omega_{k}}$ is the Dirac delta function concentrated at the frequency $\omega_{k}$, $1 \leq k \leq N$. Hence, we will show that for any test function $z(u), u \in[0,1]$,

$$
\int_{0}^{1} z(u) d\left(\sum_{k=1}^{N} I\left(\omega_{k}\right) \delta_{\omega_{k}}\right)
$$


converges to

$$
\int_{0}^{1} z(u)\left(\sum_{h \in \mathbf{Z}} E\left(X_{0} X_{h}\right) \exp (-2 \pi i h u)\right) d u
$$

when $N$ goes to infinity.

By integrating the smoothed periodogram against a test function $z(u)$, $u \in[0,1]$, and using (2.19)

$$
\begin{gathered}
\lim _{N \rightarrow \infty} \lim _{v \rightarrow \infty} \sum_{k=1}^{N} I\left(\omega_{k}\right) z\left(\frac{k}{N}\right)= \\
=\lim _{N \rightarrow \infty} \lim _{v \rightarrow \infty} \sum_{k=1}^{N}\left(\sum_{|h|<N} \sum_{j=1}^{v} c_{j} F_{\alpha}^{h}\left(c_{j}, y_{j}\right) g\left(c_{j}\right) \Delta_{j} \exp \left(-i h \frac{2 \pi k}{N}\right)\right) z\left(\frac{k}{N}\right)= \\
=\int_{0}^{1}\left[\sum_{h \in \mathbf{Z}}\left(\int_{0}^{1} x \phi\left(F_{\alpha}^{h}(x, y)\right) g(x) d x\right) \exp (-2 \pi i h u)\right] z(u) d u= \\
=\int_{0}^{1}\left(\sum_{h \in \mathbf{Z}} E\left(X_{0} X_{h}\right) \exp (-2 \pi i h u)\right) z(u) d u .
\end{gathered}
$$

Therefore, the smoothed periodogram converges in distribution sense to the spectral density function.

The property considered above in (2.20) describes a method for obtaining a good approximation to the spectral density function. This method will be explained below.

Consider $z(u)=I_{[x-\epsilon, x+\epsilon]}(u)$ for a fixed $x$ and a small fixed $\epsilon$.

From the reasoning described before, for such $z(u),(2 \epsilon)^{-1} \sum_{k=1}^{N} I\left(\omega_{k}\right) z(k / N)$ is approximately equal to

$$
\sum_{h=1-N}^{N-1} E\left(X_{0} X_{h}\right) \exp (-2 \pi i h u),
$$

if $N$ is large and $\epsilon$ small enough.

Considering now several $z_{i}(u)=I_{\left[x_{i}-\epsilon, x_{i}+\epsilon\right]}(u)$, where $x_{i}$ are equally spaced,

$$
\left[x_{1}-\epsilon, x_{1}+\epsilon\right) \cup\left[x_{2}-\epsilon, x_{2}+\epsilon\right) \cup \ldots \cup\left[x_{n}-\epsilon, x_{n}+\epsilon\right]
$$


is a partition of $[0,1]$ and applying the same reasoning to each $z_{i}(u)$, we obtain the approximate shape of the graph of

$$
\sum_{h=1-N}^{N-1} E\left(X_{0} X_{h}\right) \exp (-2 \pi i h u), u \in[0,1],
$$

as a function of $u$.

From the above expression, one can derive the approximate graph of the spectral density $f_{X}(u)$ or $f_{Z}(u)$.

The proceeding just described above is called smoothing the data (see Brockwell and Davis (1987)). For instance, if one takes a large sample $T_{\alpha}^{t}\left(x_{0}\right)$, for $1 \leq t \leq 10,000$, the periodogram is given by

$$
\begin{aligned}
I\left(\omega_{k}\right) & =N^{-1} \sum_{t=1}^{N} X_{t} \exp \left(-i \omega_{k} t\right) \sum_{s=1}^{N} X_{s} \exp \left(i \omega_{k} s\right)= \\
& =N^{-1} \sum_{t=1}^{N} \sum_{s=1}^{N} X_{s} X_{t} \exp \left(-i(t-s) \omega_{k}\right)
\end{aligned}
$$

and one can plot this real function in the interval $(0,2 \pi]$ as a function of $\omega_{k}$. This graph will show a sparse amount of data, but if one takes a partition of the interval in small intervals and takes means of this data in each small interval (also called smoothing the data), then the graph of a well defined spectral density

$$
\sum_{h=-\infty}^{\infty} E\left(X_{0} X_{h}\right) \exp (-2 \pi i h u)
$$

as described in this section will be obtained.

\section{REFERENCES}

BOGOMOLNY, E. and CARIOLI, M. (1995) Quantum Maps of Geodesic Flows on Surfaces of Constant Negative Curvature. To appear in Phy sica $D$.

BROCKWELL, P. J. and DAVIS, R. A. (1987) Time Series: Theory and ethods. New York: Springer-Verlag.

LOPES, A. and LOPES, S. (1995) Parametric Estimation and Spectral Anal ysis of Chaotic Time Series. Submitted. 
PARRY, W. and POLLICOTT, M. (1990) Zeta Functions and the Periodic Orbit Structure of Hyperbolic Dynamics. Asterisque, Vol. 187-188.

ROBINSON, C. (1995) Dynamical Systems, New York: CRC Press.

RUELLE, D. (1978) Thermodynamic Formalism, assachusetts: Addison Wesley. 
Pubicaroes do Instituto de Matematica da UFRgS

Cadernos de Matemática e Estatistica

\section{Serie A: Trabalho de Pesquisa}

1. Marcos Sebastiani Artecona - Transformation des Singularités MAP/ 89

e. Jaime Bruck Ripoll - on a Theorem of R. Langevin about Curvature and Complex singularities - MAP/89

3. Eduardo Cisneros, Miguel Ferrero e Maria Inés Gonzales - Prime Ideals of Skew Polynomial Rings and Skew Laurent Polynomial Fings - ABR 89

4. Oclide José Dotto - $\varepsilon$-Dilations - IUN 89

5. Taime Bruck Ripoll - A Characterization of Helicoids - JUN/89

6. Mark Thompson eV. B. Moscatelli - Asymptotic Distribution of Liusternik-Schnirelman Eigenvalues for Elilptic Nonlinear. Operators - JUL/89

7. Mark Thompson - The Formula of Weyl for Regions with a Self-Similar Fractal Boundary - JuL/89

8. Taime Bruck Ripoll - A Note on Compact Surfaces with Non Zero Constant Mean Curvature - OuT/89

9. Jaime Bruck Ripoll - Compact $\varepsilon$-Convex Hypersurfaces - Nov/89

10. Jandyra Maria $G$. Fachel - Coeficientes de Correlaçáo Tipo-Contigencia - IAN 90

11. Jandyra Maria G. Fachel - The Frobality of Ocurrence of Heywood Cases - IAN 90 
12. Jandyra Maria G. Fachel - Heywood Cases in Unrestricted Factor Analysis - JAN 90

13. Juilo cesar R. Claeyssen e Tereza Tsukazan de Ruiz Dynamical Solutions of Linear Matrix Diferential Equations IAN, 90

14. Maria T. Albanese - Behaviour of de Likelihood in Latent Analysis of Binary Data - AER/ 91

15. Maria T. Albanese - Measuremente of the Latent Trait Analysis of Binary Data - ABR 91

16. Maria $T$. Albanese - Adequacy of the Asymptotic Variance-Covariance Matrix Using Bootstrap Jackknife Techriques in Latent. Trait Aralysis of Binary Data - ABR/91

17. Maria T. Albanese - Latent Variuble Models for Einary Response - $\mathrm{ABE}, \mathrm{O}_{1}$

18. Mark. Thompson - Kinematic Dynamo in Random Flows - DEZ/90

19. Taime Bruck Ripoll e Marcos Sebastiani Artecona- The Generalized Map and Applications - AGO. 91

20. Jaime Bruck Ripoll, Suzana Fornari e Katia Frensel Hypersurfaces with Constant Mean Curvature in the Complex Hyperbolic Space - AO0 91

21. Suzana Fornari. E Jaime Bruck Ripoll - Stability of Compact Hypersurfaces with Constant Mean Curvature - JAN/92

ee. Marcos Sebastiani Artecona - Une Generalisation de L.Invariant de Malarange - FEV Ge

e3. Cornelis Kraaikampe Artur Lopes - The Theta Goup and the Continued Fraction with Even Furtial quotients - Mapoe 
24. Silvia Lopes - Anplitude Estimatuon un Multiple Frequency Spectrum - MAF Ge

25. Silvia Lopes e Benjamin Keden - Simusoidal Frequency Modulated Spectrum Analysis - MAF 98

26. Silvia Lopes e Eenjamin Kedem - Iteration of Mappings and Fixed Spectrum Analy=is - MAR GE

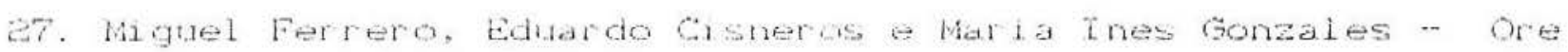
Extensions and Jarobson Pings - MAT ge

Pés. Sara C. Garmona - An Asymitolic Froblem ior a Pejction-DLtidsiri Component - ith. ge

39. Luz Fernando Carvahe da Pocha - Unigue Ergodicity ot Interval Exchange Maps - TuL ge

30. Sara C. Carnona - wave Front Propagation for a Cauchy Problem With a Fast Component - OUTAa

31. Marcos Sebastiani Artecona e Iván Fan Perez - Tntersections

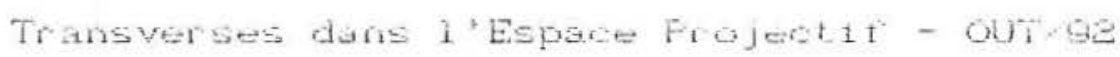

32. Miguel Ferrero - Closed Bimodules over Frime Rings: Closed Sumodules and Applications to fings Extensions - DEZ 98

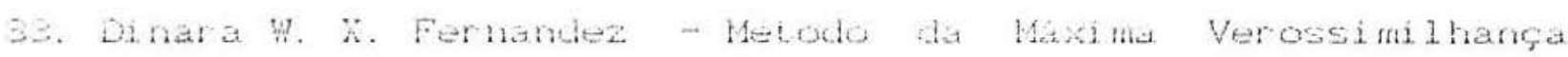
Restrita para Estimasio de Componentes de Variancia - SET/93

34. Mertin Knott e M. Ttrest Albanest - Polymiss: A Computer Progran for Fitting a One- or Two Factor Logit-Probit Latent Varlable Model to Folyoumb Dato witun observations may be inssing - Or.

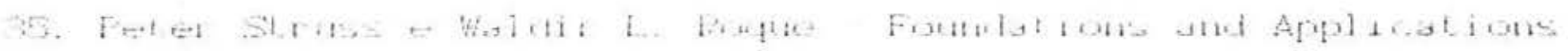

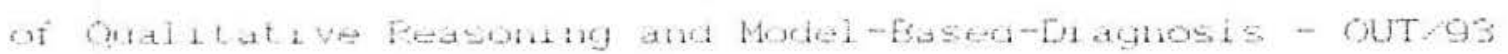


36. Edmund R. Puczylowski - on Koethe's Problem - outra3

37. Lus 6. Mendes e Marcos A. A. Eebastiani Artecona - Sur la Densite des Systemes de Ffât sans Bolution Algebrique - JAN 94

38. Artur 0. Lopes e Luz F. C. da Focha - Invariant Measures for Gauss Maps Associated with Interval Exchange Maps MAE G4

39. Artur 0. Lopes e Roberto Markatian - Open Fililards: Cantor Eels, Invariant ard Goncistionally Invariant Probabilities - A6004

40. Pierre Collet, Antono Galves $\rightarrow$ Artut D. Lopes - Maximum Likelshood and Minimum Entropy identitcation of Gramars 400,94

4h. Ondina F. Leal e Jandyra M. G. Fachel - Antropología do Corpo e Pesquisa sobre Sexualidade: Dachs Guait abivos e Tratamento Estatistaco, uma Proposta Metodologica - DEZ 94

42. Artur O. Lopes e Silvia F. C. Lopes - Farametric Estimation and Spertral Analysis oi Chaotic Time Series - Novess

43. Artur O. Lopes. Ratael R. Souza e Silva F. C. Lopes - on the Brectral Density of a Cisss of Chaotic Time Ser les - DEZ/9S 


\section{UNIERGHADE FEDERAL DO FH GEANEE DO SH WUSTIUTO DH MATEMÁRCA \\ NÚCLEO DL ATIVDADFS EXTRACURRICUL ARES}

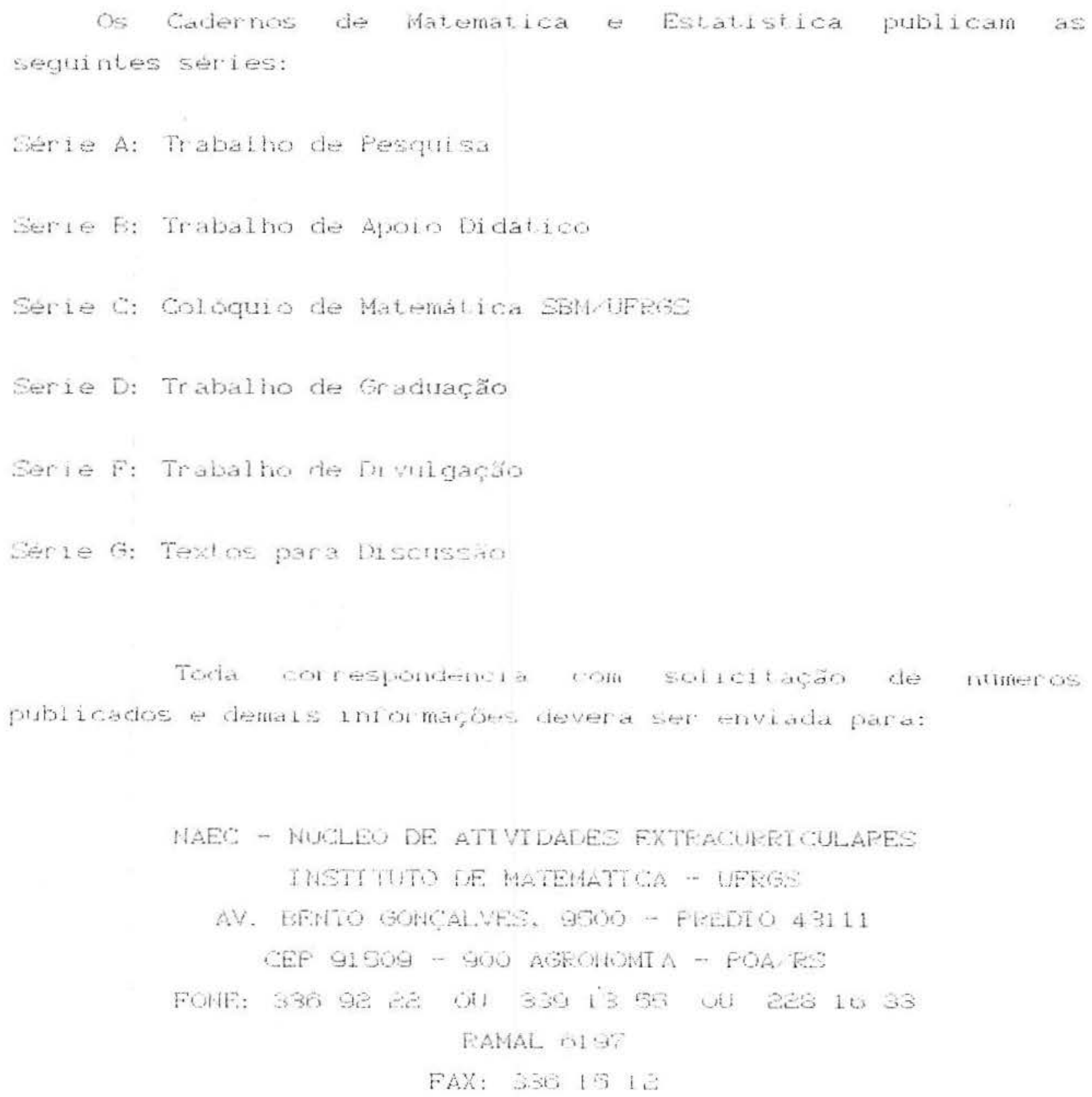

